\title{
The MDM2/MDMX/p53 axis in the adaptive stress response
}

\author{
Bing Wang ${ }^{1,2,3}$, Cody Rasmussen-Ivey ${ }^{3}$, John B. Little ${ }^{3}$, Zhi-Min Yuan ${ }^{3}$ \\ ${ }^{1}$ Key Laboratory of Space Radiobiology of Gansu Province \& Key Laboratory of Heavy Ion Radiation Biology and Medicine of Chinese Academy of \\ Sciences, Institute of Modern Physics, Chinese Academy of Sciences, Lanzhou 730000, China; ${ }^{2}$ University of Chinese Academy of Sciences, Beijing \\ 100049, China; John B. Little Center for Radiation Sciences, Harvard T. H. Chan School of Public Health, Boston, MA, USA \\ Contributions: (I) Conception and design: ZM Yuan, B Wang; (II) Administrative support: None; (III) Provision of study materials or patients: All \\ authors; (IV) Collection and assembly of data: B Wang, C Rasmussen-Ivey; (V) Data analysis and interpretation: None; (VI) Manuscript writing: All \\ authors; (VII) Final approval of manuscript: All authors. \\ Correspondence to: Zhi-Min Yuan. John B. Little Center for Radiation Sciences, Harvard T.H. Chan School of Public Health, Boston, MA 02115, \\ USA. Email: zyuan@hsph.harvard.edu.
}

\begin{abstract}
Via regulation of cellular stress responses, p53 contributes to the maintenance of homeostasis. Contrary to its well-established pro-death function, p53 is also implicated in promoting cell survival by mediating the adaptive stress response. Emerging data reveal that the adaptive stress response is coupled with p53 decline that is a prerequisite for the induction of pro-survival pathways augmenting cell fitness. However, if the adaptive stress responses persist or become chronic, the sustained p53 downregulation would result in a permanent loss of p53 function and p53-dependent homeostasis. The available information suggests a model in which cells respond to different levels of stress by governing the activity and abundance of p53 that, in turn, determines the cell fate dependent on not only the intensity but also the duration of stress.
\end{abstract}

Keywords: The MDM2/MDMX/p53 axis; mild stress; adaptive stress response; cell fate

Submitted Nov 17, 2019. Accepted for publication Dec 20, 2019.

doi: $10.21037 /$ tcr.2019.12.89

View this article at: http://dx.doi.org/10.21037/tcr.2019.12.89

\section{Introduction}

A proper stress response is critical for the maintenance of homeostasis. To preserve their fitness in an energetically sensible way, cells must constantly make precise decisions that range between survival and death according to the level of stress. To cope with harsh stresses that can potentially threaten their genome stability and the integrity of other essential structures, cells must sense cellular damage and promptly activate an appropriate response, such as cell cycle arrest, DNA repair, or, if the damage is unrepairable, apoptosis. However, living cells or organisms are also exposed to temporary and modest levels of stress that are more often encountered in our living environment. In response to such transient and mild stresses, arresting cell cycle progression or inducing cell death would be insensible. In this context, cells have to calibrate their stress response to the perturbation based on the level of stress.
Relative to severe stress, the cellular response to mild stress is less well characterized.

As a protein extremely responsive to stress, p53 plays a central role in the maintenance of homeostasis by regulating a number of cellular pathways in response to both endogenous and exogenous stress cues (1). Under the condition of severe stress, $\mathrm{p} 53$ is robustly activated, inducing the expression of a host of genes whose products mediate either apoptosis or senescence to eliminate irreparably damaged cells, which is critical for protecting organismal fidelity. However, growing evidence also implicates p53 in promoting adaptation and survival responses to mild stress in support of organismal fitness. It appears that $\mathrm{p} 53$ is a multifaceted stress-responsive protein that can sometimes mediate seemingly opposing effects on biological pathways, such as the pro-survival activity, which is in clear conflict with p53's canonical pro-death functions. While the 
intricate stress responses mediated by $\mathrm{p} 53$ are likely context dependent, the role of p53 in cellular response to mild stress is much understudied relative to severe stress.

To understand the p53 response to stress, it is of importance to appreciate how p53 is regulated. Among several other players, MDM2 and MDMX are the two essential negative regulators of $\mathrm{p} 53$, as convincingly demonstrated by genetic studies where knock out either MDM2 or MDMX resulted in p53-dependent embryonic lethality (2-5). However, it was initially unclear why these two p53 inhibitors could not compensate for the loss of each other since in MDM2 knockout mice the MDMX expression was intact and vice versa. Later studies performed independently by Huang et al. and Pant et al. demonstrated that disassociation of the MDM2/MDMX complex in mice is associated with embryonic lethality. Of importance is that this lethality is completely rescued by concomitant deletion of $\mathrm{p} 53$, establishing that an integral MDM2/MDMX complex is essential for effective p53 control, at least during the embryonic stage $(6,7)$. Via their respective RING domain, MDM2 and MDMX bind to each other to form a complex (8). Structural studies with crystal and NMR analysis have revealed that the heterocomplex of MDM2/MDMX is more energetically favorable than the MDM2 homocomplex (9). In support of the heterocomplex being more stable, the MDM2/ MDMX complex is the dominant form found in cells. Upon binding to MDMX, MDM2 becomes more stable and functions as a better E3 ligase for p53. In the absence of MDM2 expression, the MDMX protein is localized in the cytoplasm due to a lack of the nuclear localization sequence. As a result, MDMX alone is unable to bind to and inhibit p53 as the latter exists primarily in the nucleus. Binding to MDM2 however brings MDMX into the nucleus where the complex can effectively inhibit p53 activity. The mutual dependency between MDM2 and MDMX underpins the requirement of the intact MDM2/MDMX complex in p53 control (10). In line with this model, various stress signals converge on the MDM2/MDMX complex to modulate p53 activity. For instance, DNA damage induced MDMX phosphorylation by ATM and Chk2 stimulates MDM2dependent MDMX ubiquitination/degradation resulting in reduced MDM2/MDMX complex levels and subsequent p53 activation (11-13). Oncogenic stresses also target MDMX for phosphorylation, which however is associated with enhanced stability of the MDM2/MDMX complex and subsequently increased p53 degradation (14-16). Ample information indicates that the MDM2/MDMX complex integrates diverse intra- and extra-cellular stress signals regulating $\mathrm{p} 53$ response to perturbations of cellular homeostasis (17). Given that the severe stress-induced p53 response is fairly well understood, we sought to focus our discussion on the p53-mediated cellular response to mild stress.

\section{Evidence supporting p53-dependent response to mild stress}

Exposure of cells to high doses of ionizing radiation (IR) induces considerable DNA damage resulting in injury that is associated with robust $\mathrm{p} 53$ activation, whereas low doses of radiation (LDR) induces an adaptive response, as reflected by enhanced survival. Such a dose-dependent stress response is also widely observed with many other different types of stress (18). We thus choose LDR as a representative to discuss the $\mathrm{p} 53$ response to mild stress. Other types of stress will be included when data are available. We define LDR as the dose of radiation below the threshold able to induce detectable DNA damage. Because the direct biological effects of LDR are relatively subtle and difficult to measure, the effects of LDR are often investigated in the context of the radioadaptive response, which is characterized as an enhanced resistance to high dose radiation-induced harmful effects by pretreatment with LDR. This adaptive response is in fact part of a general cellular response to stress that is evolutionally conserved and has been observed from single cell organism such as yeast to mammalian cells (18). Various readouts including DNA damage, chromosomal aberration, cell death, mutagenesis and among others, have been used to determine the radioadaptive response. In the context of DNA damage, it was reported that pretreatment of C57BL/6N mice with $500 \mathrm{mGy}$ during a span of 23 days considerably reduced a challenge dose of radiation-induced DNA damage in the spleen, a very radiosensitive tissue (19). The radioadaptive response was also reported with an end point of cancer development. Treatment of Swiss mice with a priming dose of $10 \mathrm{mGy}$ daily for 5 or 10 days was associated with a significant decrease in the lymphoma incidence induced by $2 \mathrm{~Gy}$ of irradiation (20). A critical contribution of $\mathrm{p} 53$ to the radioadaptive response was first reported by Horie and his colleagues. Using $\mathrm{p} 53^{-/-}, \mathrm{p} 53^{+/-}$ and $\mathrm{p} 53^{+/+}$mice, the authors showed that pretreatment of mice with a priming dose of irradiation ( $0.45 \mathrm{~Gy})$ induced marked radioadaptive response in wildtype mice but not $\mathrm{p} 53^{-/-}$mice. Of note is that $\mathrm{p} 53^{+/-}$mice exhibited an intermediate radioadaptive response, consistent with a p53 
gene-dosage dependent radioadaptive response (21). A critical role of $\mathrm{p} 53$ in mediating the radioadaptive response was further corroborated by the study from Jiang et al. who reported that the radioadaptive response was specific to normal but not tumor cells (22).

Although animal studies provided solid evidence to support the concept of radioadaptive response, it remained a topic of intensive debate because many observations derived from animal experiments could not be recapitulated in cellbased in vitro studies. While there might be a number of factors contributing to this disparity, it was found that the difference in oxygen concentration between in vivo and in vitro conditions played a major role (23). In the standard tissue culture conditions, the oxygen pressure is around 20 $21 \%$ whereas the $\mathrm{O}_{2}$ concentration in vivo is approximately $5 \%$. As a result, cells cultured in vitro experience an excess amount of oxygen often causing oxidative stress. Such stressful in vitro culture conditions would render cells insensitive to LDR treatment because LDR-induced effects are largely mediated by a modest increase in ROS. The small amount of ROS is readily masked under the in vitro tissue culture condition due to the high $\mathrm{O}_{2}$ concentration. Indeed, in contrast with the standard tissue culture condition, cells cultured under the physiological $\mathrm{O}_{2}$ concentration (3-5\%) exhibited comparable radioadaptive responses to those observed in vivo. Pretreatment of cells with LDR induced significant resistance to subsequent challenging dose IR-induced DNA damage. Additional evidence supporting a crucial role of ROS came from the observation that treatment of cells with $\mathrm{N}$-acetyl cysteine, an antioxidant, abrogated the radioadaptive response. The importance of physiological oxygen concentration may have very broad implication in the study of cellular response to stress in general and to the moderate level of stress in particular.

\section{Mechanisms behind p53-mediated radioadaptive response}

Although p 53 was reported to play a role in the radioadaptive response, the underlying mechanism remained unclear, which to a large extent is due to the fact that LDR typically induces little DNA damage and dose not overtly activate p53. Indeed, it was found that p53 was infect downregulated in LDR-treated cells (23). Remarkably, this p53 decline was essential for inducing the radioadaptive response. LDRinduced p53 downregulation was mediated by ROS, which stimulates p38, a stress-responsive kinase. Upon activation, p38 phosphorylates MDMX resulting in an increase in the stability and activity of the MDM2/MDMX complex. As a consequence, MDM2/MDMX-mediated p53 turnover is increased resulting in a reduction in p53 levels (16).

The association of $\mathrm{p} 53$ decline with LDR-induced survival is not in conflict with the canonical prodeath function of $\mathrm{p} 53$. Interestingly, LDR-induced $\mathrm{p} 53$ downregulation was accompanied with an induction of HIF1, a master transcription factor critical for metabolic regulation (24), providing an interesting link to metabolism. In accordance, the radioadaptive response was associated with a metabolic switch from catabolic oxidative phosphorylation to anabolic glycolysis (23). This metabolic response is analogous to the Warburg effect, a phenomenon initially thought as a unique metabolic feature of transformed cells. However, growing evidence indicates that the Warburg metabolism is the anabolic program necessary for supporting cell growth and proliferation shared by most growing cells (25). In the context of the radioadaptive response, HIF1-mediated anabolic metabolism is essential for LDR-induced survival, representing a metabolic mechanism behind the radioadaptive response. Such anabolic metabolism-mediated adaptive response was also observed when cells were treated with low levels of arsenic, $\mathrm{H}_{2} \mathrm{O}_{2}$ and among others (26), implicating the metabolic response as a common mechanism underlying the adaptive stress response. The available information implicates that $\mathrm{p} 53$ under physiological conditions keeps anabolic metabolism in check. Either an increase or decrease in the p53 level would impact cellular metabolic programs. In this context, a low level of stress induces p53 decline enabling the induction of anabolic metabolism to augment the adaptive response. In the event of severe stress or very high of an increase in ROS, p53 is activated, resulting in the induction of either cell cycle arrest, senescence or apoptosis where the anabolic metabolism is suppressed (27). In addition to the stress response, p53 downregulation was reported to be required for the induction of anabolic metabolism to support $\mathrm{T}$ cell activation and proliferation (28). It was also shown that $\mathrm{p} 53$ reduction is critical to allow mTOR-mediated anabolic metabolic pathway augmenting cell fitness (29). Further studies will be necessary to understand how 553 restrains anabolic metabolism to preserve homeostasis.

In summary, the adaptive stress response, if transient or temporary, is largely protective or beneficial because it induces the cellular pathways that can not only minimize potential damage but also enhance robustness. However, 
if stress conditions persist or become chronic, the adaptive response could become detrimental resulting in pathological consequences. With the finding that the adaptive response is associated a decline in 553 levels, it becomes clear why prolonged or chronic adaptive stress response is largely harmful because the sustained $\mathrm{p} 53$ downregulation would cause a permanent loss of $\mathrm{p} 53$-dependent homeostasis leading to pathological outcomes. The available information suggests a model in which cells respond to different levels of stress by governing the activity and abundance of p53 that, in turn, determines the cell fate dependent on not only the intensity but also the duration of stress.

\section{Acknowledgments}

Funding: This work was supported in part by the Morningside Foundation, the Zhu Fund and grants from the National Cancer Institute at the National Institutes of Health (R01CA233558, R01CA167814, and R01CA125144).

\section{Footnote}

Conflicts of Interest: All authors have completed the ICMJE uniform disclosure form (available at http://dx.doi. org/10.21037/tcr.2019.12.89). ZMY serves as an unpaid editorial board member of Translational Cancer Research from Jun 2019 to May 2021. The other authors have no conflicts of interest to declare.

Ethical Statement: The authors are accountable for all aspects of the work in ensuring that questions related to the accuracy or integrity of any part of the work are appropriately investigated and resolved.

Open Access Statement: This is an Open Access article distributed in accordance with the Creative Commons Attribution-NonCommercial-NoDerivs 4.0 International License (CC BY-NC-ND 4.0), which permits the noncommercial replication and distribution of the article with the strict proviso that no changes or edits are made and the original work is properly cited (including links to both the formal publication through the relevant DOI and the license). See: https://creativecommons.org/licenses/by-nc-nd/4.0/.

\section{References}

1. Kruiswijk F, Labuschagne CF, Vousden KH. p53 in survival, death and metabolic health: a lifeguard with a licence to kill. Nat Rev Mol Cell Biol 2015;16:393-405.

2. Jones SN, Roe AE, Donehower LA, et al. Rescue of embryonic lethality in Mdm2-deficient mice by absence of p53. Nature 1995;378:206-8.

3. Montes de Oca Luna R, Wagner DS, Lozano G. Rescue of early embryonic lethality in mdm2-deficient mice by deletion of p53. Nature 1995;378:203-6.

4. Parant J, Chavez-Reyes A, Little NA, et al. Rescue of embryonic lethality in Mdm4-null mice by loss of Trp53 suggests a nonoverlapping pathway with MDM2 to regulate p53. Nat Genet 2001;29:92-5.

5. Finch RA, Donoviel DB, Potter D, et al. MDMX is a negative regulator of $\mathrm{p} 53$ activity in vivo. Cancer Res 2002;62:3221-5.

6. Huang L, Yan Z, Liao X, et al. The $\mathrm{p} 53$ inhibitors MDM2/ MDMX complex is required for control of p53 activity in vivo. Proc Natl Acad Sci USA 2011;108:12001-6.

7. Pant $\mathrm{V}$, Xiong $\mathrm{S}$, Iwakuma $\mathrm{T}$, et al. Heterodimerization of $M d \mathrm{~m} 2$ and $M d m 4$ is critical for regulating p 53 activity during embryogenesis but dispensable for $\mathrm{p} 53$ and $\mathrm{Mdm} 2$ stability. Proc Natl Acad Sci USA 2011;108:11995-2000.

8. Kawai H, Lopez-Pajares V, Kim MM, et al. RING domain-mediated interaction is a requirement for MDM2's E3 ligase activity. Cancer Res 2007;67:6026-30.

9. Linke K, Mace PD, Smith CA, et al. Structure of the MDM2/MDMX RING domain heterodimer reveals dimerization is required for their ubiquitylation in trans. Cell Death Differ 2008;15:841-8.

10. Gu J, Kawai H, Nie L, Kitao H, et al. Mutual dependence of MDM2 and MDMX in their functional inactivation of p53. J Biol Chem 2002;277:19251-4.

11. Kawai H, Wiederschain D, Kitao H, et al. DNA damageinduced MDMX degradation is mediated by MDM2. J Biol Chem 2003;278:45946-53.

12. Chen J. The roles of MDM2 and MDMX phosphorylation in stress signaling to p53. Genes Cancer 2012;3:274-82.

13. Li J, Kurokawa M. Regulation of MDM2 stability after DNA damage. J Cell Physiol 2015;230:2318-27.

14. Lopez-Pajares V, Kim MM, Yuan ZM. Phosphorylation of MDMX mediated by Akt leads to stabilization and induces 14-3-3 binding. J Biol Chem 2008;283:13707-13.

15. Gerarduzzi C, de Polo A, Liu XS, et al. Human epidermal growth factor receptor 4 (Her4) Suppresses 553 Protein via Targeting the MDMX-MDM2 Protein Complex: Implication Of A Novel MDMX Ser-314 Phosphosite. J Biol Chem 2016;291:25937-49.

16. de Polo A, Luo Z, Gerarduzzi C, et al. AXL receptor 
signaling suppresses p53 in melanoma through stabilization of the MDMX-MDM2 complex. J Mol Cell Biol 2017;9:154-65.

17. Wade M, Li YC, Wahl GM. MDM2, MDMX and p53 in oncogenesis and cancer therapy. Nat Rev Cancer 2013;13:83-96.

18. Calabrese EJ, Dhawan G, Kapoor R, et al. HORMESIS: A Fundamental Concept with Widespread Biological and Biomedical Applications. Gerontology 2016;62:530-5.

19. Otsuka K, Koana T, Tauchi H, et al. Activation of antioxidative enzymes induced by low-dose-rate wholebody gamma irradiation: adaptive response in terms of initial DNA damage. Radiat Res 2006;166:474-8.

20. Bhattacharjee D. Role of radioadaptation on radiationinduced thymic lymphoma in mice. Mutat Res 1996;358:231-5.

21. Horie K, Kubo K, Yonezawa M. p53 dependency of radioadaptive responses in endogenous spleen colonies and peripheral blood-cell counts in C57BL mice. J Radiat Res 2002;43:353-60.

22. Jiang H, Li W, Li X, et al. Low-dose radiation induces adaptive response in normal cells, but not in tumor cells: in vitro and in vivo studies. J Radiat Res 2008;49:219-30.

Cite this article as: Wang B, Rasmussen-Ivey C, Little JB, Yuan ZM. The MDM2/MDMX/p53 axis in the adaptive stress response. Transl Cancer Res 2020;9(3):1993-1997. doi: 10.21037/tcr.2019.12.89
23. Lall R, Ganapathy S, Yang M, et al. Low-dose radiation exposure induces a HIF-1-mediated adaptive and protective metabolic response. Cell Death Differ 2014;21:836-44.

24. Amelio I, Melino G. The p53 family and the hypoxiainducible factors (HIFs): determinants of cancer progression. Trends Biochem Sci 2015;40:425-34.

25. Wang A, Luan HH, Medzhitov R. An evolutionary perspective on immunometabolism. Science 2019. doi: 10.1126/science.aar3932.

26. Gong L, Pan X, Abali GK, Little JB, Yuan ZM. Functional interplay between $\mathrm{p} 53$ and $\Delta 133 \mathrm{p} 53$ in adaptive stress response. Cell Death Differ 2019. [Epub ahead of print].

27. Vousden KH, Ryan KM. p53 and metabolism. Nat Rev Cancer 2009;9:691-700.

28. Watanabe M, Moon KD, Vacchio MS, et al. Downmodulation of tumor suppressor $\mathrm{p} 53$ by $\mathrm{T}$ cell receptor signaling is critical for antigen-specific CD4(+) $\mathrm{T}$ cell responses. Immunity 2014;40:681-91.

29. Bowling S, Di Gregorio A, Sancho M, et al. P53 and mTOR signaling determine fitness selection through cell competition during early mouse embryonic development. Nat Commun 2018;9:1763. 